\title{
Space Boundary Topology Simplification for Building Energy Performance Simulation Speedup
}

\author{
Georgios N. Lilis ${ }^{1}$, Georgios I. Giannakis ${ }^{1}$, Kyriakos Katsigarakis ${ }^{1}$, Dimitrios Rovas ${ }^{2}$ \\ ${ }^{1}$ School of Production Engineering \& Management, Technical University of Crete, Greece \\ ${ }^{2}$ Institute for Environmental Design and Engineering, University College London, UK
}

\begin{abstract}
In recent studies, methodologies for automated Building Energy Performance simulation model generation, utilizing Building Information Model data, have received considerable attention. In most cases, each space of a building is treated as a thermal zone, and each $2^{\text {nd }}$-level space boundary as an inter-zone boundary surface. This results in increased computational effort that scales with the number of zones and surfaces, but without necessarily improved simulation accuracy. Geometry simplification can be quite beneficial to reduce complexity; however, its effectiveness relies on the modeler experience and the building's geometric characteristics. In prior work, zone merging approaches have been investigated but the topic of geometric simplification has remained relatively unexplored. In this work, an algorithmic process is introduced for simplification of the $2^{\text {nd }}$-level space boundary topology, and consequently reduction of the number of surfaces.
\end{abstract}

\section{Introduction}

According to the Building Energy Performance Simulation (BEPS) 3D zonal-models definition, a building consists of thermal zones, coupled with each other and with the environment. A thermal zone consists of an air volume of uniform temperature distribution, its bounding surfaces and elements contained inside this volume. The basis for the zone air temperature estimation in BEPS, is the formulation of energy and moisture balances for the zone air and the solution of the resulting ordinary differential equations. The computational effort behind these operations is proportional to the total number of building thermal zones and their boundary surfaces, which depends on the building geometry and its space topology.

In recent studies, development of methodologies for automated BEPS zonal-models generation, utilizing Building Information Model (BIM) data, has received considerable attention (Andriamamonjy et al., 2018; Thorade et al., 2015; Wimmer et al., 2015; Giannakis et al., 2015). Although these methodologies make the BEPS model generation process much more expedi- ent, they suffer from design and exporter errors during the BIM generation stage and the simulation of the generated model is time-consuming since its geometry and zoning approximations are considered of high detail, but without necessarily improved simulation accuracy: each space of a building is treated as a thermal zone and the $2^{\text {nd }}$-level space boundaries (surfaces of thermal exchange among building spaces and the environment) (Weise et al., 2011), as inter-zone boundary surfaces. This results in increased computational effort, since the simulation process is predominantly slowed down by the increased number of zones and their bounding surfaces. Consequently, reduction of the simulation run time can be achieved by either decreasing the number of thermal zones or their boundary surfaces. Furthermore, an increased geometrical complexity arising from an increased number of zones in a BEPS model, adds difficulty in the interpretation and the post processing of the simulation results. Consequently, zone number reduction methods appears to be a reasonable solution to facilitate these processes.

Concerning the zone number reduction methods, in prior work, manual (ASHRAE 90.1, 2010) and automated (Giannakis et al., 2017; Georgescu and Mezić, 2015) zone merging approaches have been investigated. Most of these methods are based on spacesto-zones grouping rules leaving the geometric content of BEPS models intact and as a result the topic of an geometric simplification of BEPS models using zone reduction has remained relatively unexplored. To this direction, geometry simplification attempts by manually editing the generated BEPS input data file can be quite beneficial to reduce complexity; however, their effectiveness relies on the modeler experience and the building's geometric characteristics (Giannakis et al., 2013).

In the present work, a novel geometric simplification algorithm is introduced to support automated zoning reduction approaches as the ones presented in (Giannakis et al., 2017). The proposed algorithm is essentially a transformation process from the buildings' $2^{\text {nd }}-$ level space boundary surface topology (Weise et al., 2011) (illustrated by the floor plan in 
part I of Figure 1) to a simplified thermal zone boundary surface topology (illustrated by the floor plan in part III of Figure 1)), given a spaces-to-zones grouping rule. In general, a thermal zone boundary surface topology constitutes the geometric core of every BEPS model and consists of the thermal energy flow surfaces among the thermal zones of the building and their environment air or ground.

Initially, when no zone reduction is applied the building spaces are treated as individual thermal zones, while the thermal energy flow surfaces are the $2^{\text {nd }}$ level space boundary surfaces (Weise et al., 2011), characterized by inside and outside (if exists) relating space indexes, as illustrated by the table of part I of Figure 1. This $2^{\text {nd }}$-level space boundary surface topology, along with a spaces-to-zones grouping rule, constitute the input of the introduced algorithm. As an intermediate step, the algorithm relies on the input spaces-to-zones grouping rule, to update the relating space indexes of the $2^{\text {nd }}$-level space boundary surfaces according to their relating zone index, as the table in part II of Figure 1 indicates with dashed rectangles. Finally, as a last step, the algorithm merges multiple coplanar and adjacent space boundary surfaces with the same updated relating zone indexes and the same related building elements, into fewer thermal boundary surfaces, as illustrated in part III of Figure 1.

In short, the reduction in the total number of thermal boundary surfaces achieved by the surface merging and the reduction in the total number of zones achieved by the spaces-to-zones grouping, has a combined effect in reducing the total simulation run time. This reduction can be justified by the reduction in the number of: (a) the differential equations solutions (which is proportional to the number of thermal zones) solved by the simulation program; and (b) the respective boundary condition evaluations (which is proportional to the number of thermal boundary surfaces) performed by the simulation routine.

To introduce the proposed algorithm its input data prerequisites are presented first, followed by analytic description of its steps. Finally, to demonstrate the achieved simulation run time reduction without significant loss of accuracy in the simulation results, comparisons between full and simplified model simulations on a demonstration building are performed.

\section{Proposed algorithm}

From a broad perspective, the simplification algorithm can be considered as a space boundary topology transformation process in which, the initial $2^{\text {nd }}$-level space boundary surfaces are merged (transformed) to zone boundary surfaces based on a spaces-to-zones grouping rule. Conceptually, the algorithm can be divided into four sequential stages, as displayed in the process diagram of Figure 2: (a) surface re-indexing; (b) intermediate surface calculation; (c) surface merg-

I. Space boundary topology

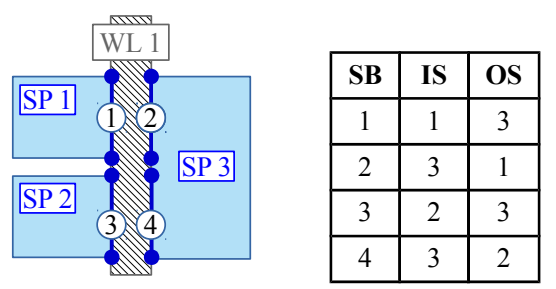

\section{Space to thermal zone boundary surface re-indexing}

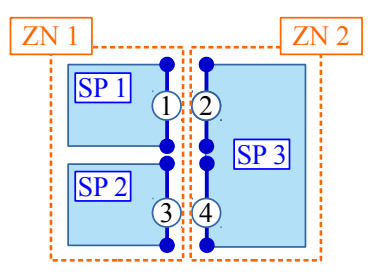

III. Thermal zone boundary surface topology

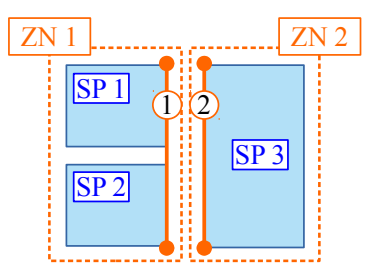

\begin{tabular}{|c|l|c|l|}
\hline SB & Space Boundary & ZB & Zone Boundary \\
\hline IS & Inner Space & IZ & Inner Zone \\
\hline OS & Outer Space & OZ & Outer Zone \\
\hline$\bullet \bullet$ & Space / Zone Boundary \\
\hline$\bullet$ & Merged Zone Boundary \\
\hline
\end{tabular}

Figure 1: Illustration of the application of: (II) space to thermal zone boundary surface re-indexing process and (III) boundary surface merging process, on a space boundary surface topology (I).

ing; and (d) zone boundary projection and intersection. These stages are described thoroughly in the following sections.

\section{Data requirements}

The proposed algorithm has two main input data requirements: (a) the $2^{\text {nd }}$-level space boundary surface data obtained from the IFC file that is used for the BEPS input data file generation, where each space is treated as an individual thermal zone (such topology is obtained by the use of tools such as CBIP (Lilis et al., 2017) or the Space Boundary Tool Rose and Bazjanac (2015)); and (b) the zones definition as groups of (or isolated) spaces (spaces-to-zones grouping).

\section{Surface re-indexing}

Every $2^{\text {nd }}$-level space boundary surface of type $2 \mathrm{~A}$ can be characterized by two space indexes, if it is internal, or by single space index, if it is external. If 


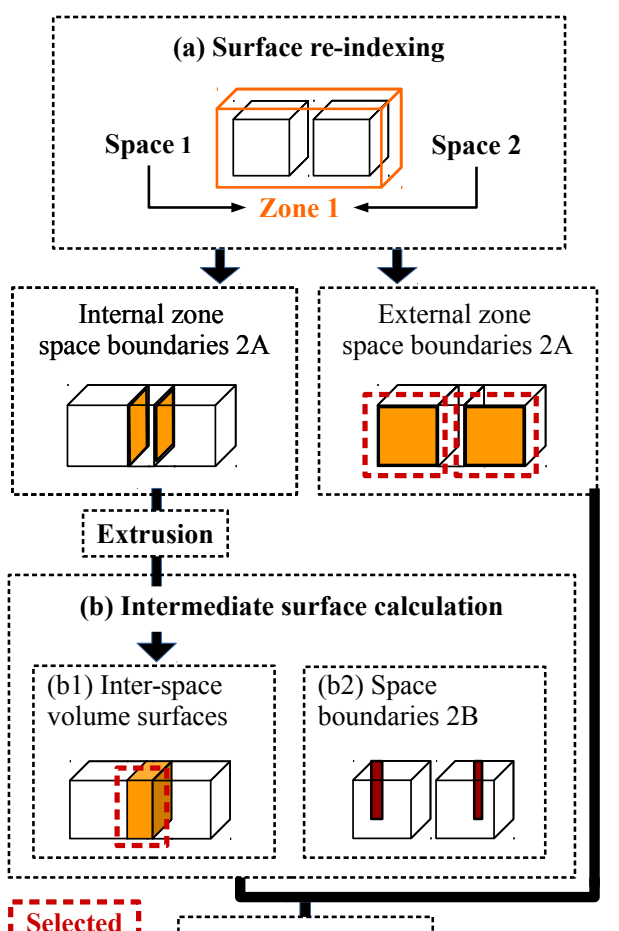

termediate surfaces) consisting of two subsets: (b1) the inter-space volume surfaces (see (b1) block of Figure 2) and (b2) the set of $2^{\text {nd }}$-level (type 2B) (Weise et al., 2011) space boundary surfaces of the spaces belonging to the same zone (see Figure 4). The surfaces of these two subsets will be used for surface merging in stage c (according to Figure 2) and are obtained as described next.

b1. Inter-space volume surfaces: The inter-space volume surfaces of a zone are the boundary representation (B-rep) surfaces of the volumes among the spaces that belong to this zone. These surfaces are obtained by an extrusion process applied on pairs of space boundary surfaces of type $2 \mathrm{~A}$ facing to each other and belonging to different spaces of the same zone (see Figure 3). During this extrusion process for every surface pair, a set of boundary representation surfaces of an inter-space volume is obtained (B-rep set). This B-rep set is initialized with one of the surfaces of the pair (selected surface).
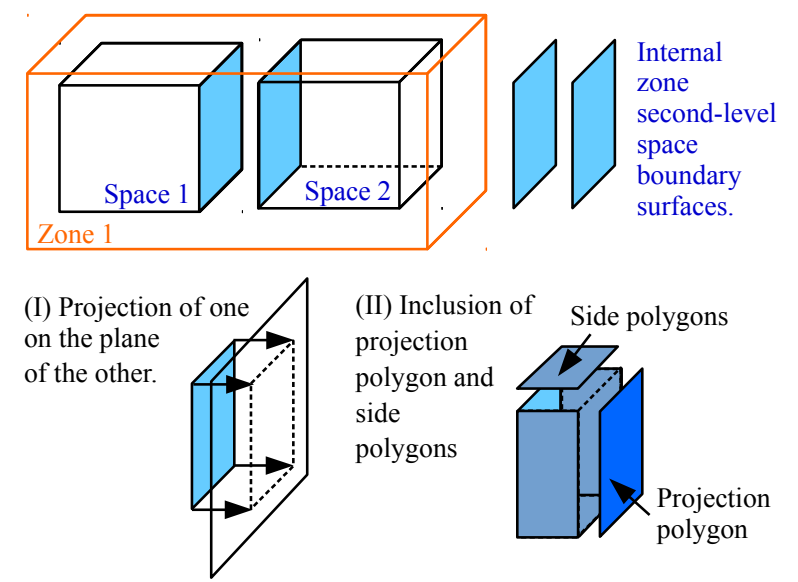

Figure 2: Algorithm overview.

the space boundary is internal, the first index refers to its relating space, while the second index refers to the relating space of its corresponding boundary. If the space boundary is external, the space index refers to its relating space. When multiple spaces are grouped to a specific zone, the space indexes of their space boundary surfaces are updated with the zone index values. This process is defined as spaces-to-zones surface re-indexing. For instance, if two space boundary surfaces have different relating spaces, which belong to the same zone, their space index values are updated with the new common zone index value. Such a re-indexing process is illustrated in part II of Figure 1 , for the boundary surfaces 1 and 3 that belong to zone 1 , and for the boundary surfaces 2 and 4 that belong to zone 2. This surface re-indexing process, used in (Giannakis et al., 2017), leaves the geometry of the space boundary surfaces and their total number intact.

\section{Intermediate surface calculation}

This stage involves all the necessary operations to determine a set of surfaces for each zone (named in-

Figure 3: Extrusion process for the generation of the boundary representation surfaces of inter-space volumes.

Then, as presented in Figure 3, the extrusion process populates the rest of the B-rep set in two stages: (I) by projecting the selected surface on the plane of the other surface of the pair and including the projection polygon to the B-rep set and (II) by generating and including in the B-rep set, the side surfaces of the solid formed between the selected surface and its projection from stage I (illustrated in Figure 3).

b2. Space boundary surfaces of type 2B: A type $2 \mathrm{~B}$ space boundary surface is illustrated with purple color in Figure 4. The type 2B $2^{\text {nd }}$-level space boundary surfaces of a specific zone are all the $2^{\text {nd }}$ level 2B boundary surfaces (Weise et al., 2011) of the spaces that are grouped to this zone.

\section{Surface merging}

In the surface merging stage, a set of zone boundary surfaces for each zone is obtained from the intermediate surface set of the zone (set A) and a set of type $2 \mathrm{~A}$ space boundary surfaces (set B) which have the 


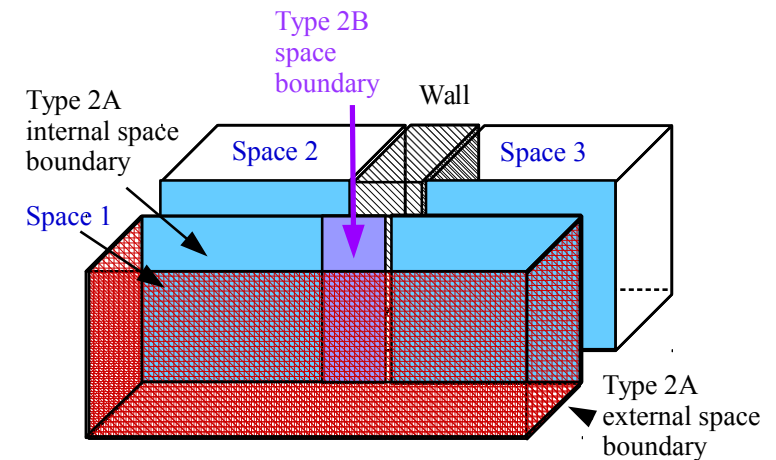

Figure 4: Illustration of a type 2B space boundary surface on a single space volume.

following characteristics: its relating space belong to the zone and the relating space of its corresponding boundary (if exists) belong to another zone. Selected coplanar space boundary surfaces from set B, which have the same relating space and their corresponding boundary surfaces (if exist) have also the same relating space are unified or merged together with coplanar intermediate surfaces from set A. Finally, during this process, each internal space boundary of type $2 \mathrm{~A}$ and its corresponding boundary, which have relating spaces belonging to the same zone are removed. Examples of coplanar adjacent surfaces which are merged during this stage are indicated with dashed boxes in Figure 2. The unification is implemented using the polygon union operations of (Vatti, 1992). With this unification process the number of the external with respect to the zone space boundaries of type $2 \mathrm{~A}$ is reduced as multiple small coplanar surfaces are fused into fewer but larger surfaces. Finally, to eliminate possible surface artifacts, these merged zonal boundary surfaces, require further processing operations, as described next.

\section{Zone boundary projection}

During this last processing stage the zone boundary surfaces obtained from the previous stage, which belong to different zones and face each other are projected on one another and the projection polygons are intersected with the original surface polygons. Finally, the initial zone boundary surfaces are replaced by the intersection polygons. The whole process is illustrated in Figure 5.

As illustrated in Figure 6, the zone boundary projection is required since it removes surface remnants caused by the surface merging, applied in the previous stage.

\section{Application}

The algorithm is demonstrated on an imaginary twostory office building depicted in Figure 7 (part I), in which the internal space volumes of the ground and first floor are not vertically aligned (part II). A slight offset was defined to demonstrate the generality and

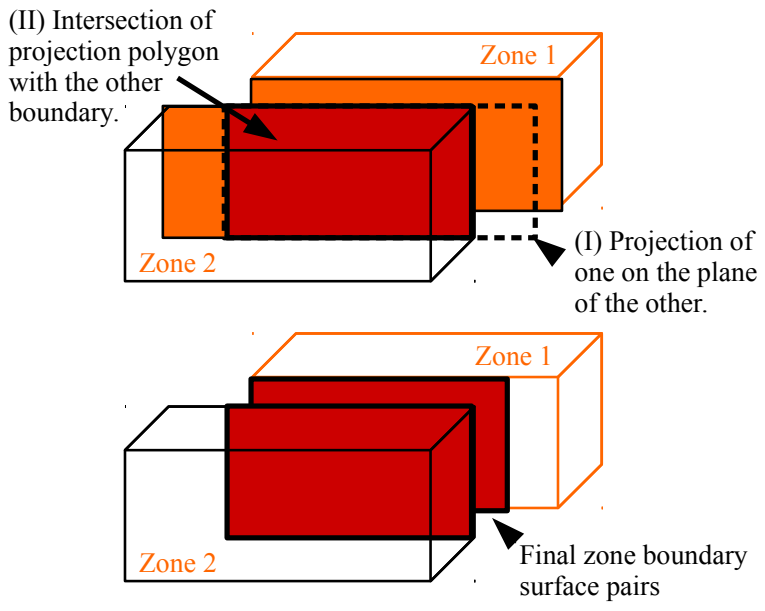

Figure 5: Illustration of projection and intersection operations on opposite zone boundary surface pairs.

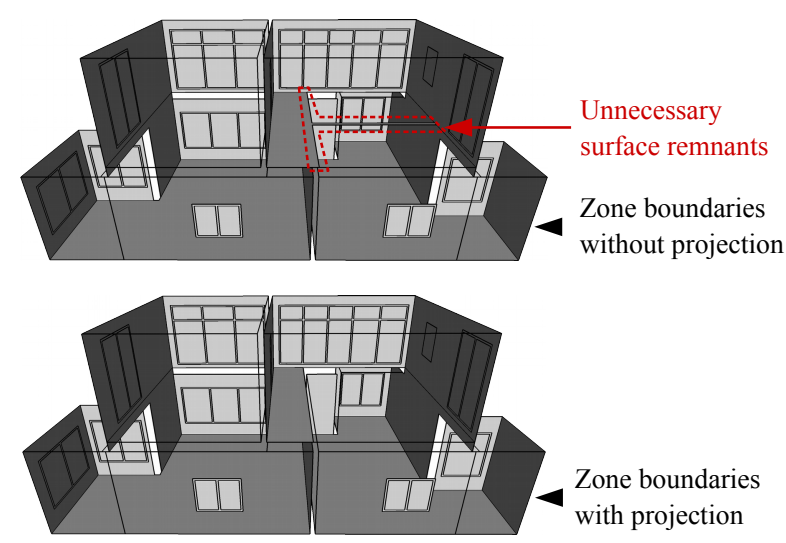

Figure 6: Unnecessary surface remnants removed by zone boundary projection.

the ability of the introduced algorithm in producing complex inter-space volume spaces (part b1 in Figure 2).

For this imaginary building two application scenarios were investigated. In the first scenario (denoted as EW), the building spaces are partitioned vertically forming two zones with east and west orientation (Zone 1: Spaces 1,2 and 5-Zone 2: Spaces 3,4 and $6)$. The space volumes of each zone for this scenario are displayed in parts IA and IC of Figure 8. In the second scenario (denoted as GF), the building spaces are partitioned forming two zones: one in the ground floor and the other in the first floor (Zone 1: Spaces 1,2,3 and 4 - Zone 2: Spaces 5 and 6 ). The space volumes of each zone for this scenario are displayed in parts IIA and IIB of Figure 8.

As parts IB-ID and IIB-IID of Figure 8 depict, the algorithm produces correctly the inter-space volume surfaces for the examined scenarios.

During the merging stage the external space boundaries referring to different construction elements belonging to the same zone are not merged. 


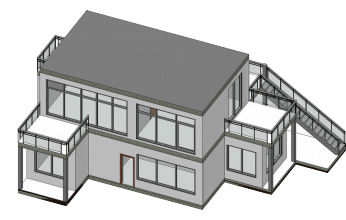

I. Building geometry

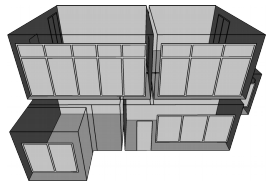

III. Space boundary topology (front view)

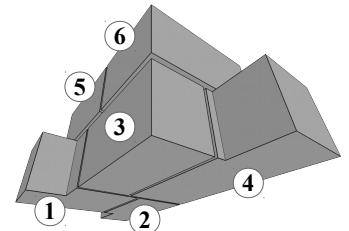

III. Building space volumes

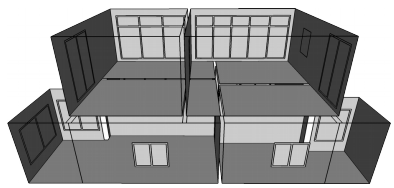

IV. Space boundary topology (rear view)
Figure 7: Demonstration building and its space volume and space boundary topology (surface culling has been applied to render the building interior visible).

The thermal boundary surface calculated by the introduced algorithm for the two examined space partition scenarios (EW and GF) on the demonstration building, are displayed in 9 . For the EW partition, as it is presented in parts IA and IB in Figure 9, all the internal space boundaries related to inner wall partitions on the ground floor having east west orientation are removed and the space boundaries on the first floor among the two zones slab are merged into one thermal zone surface boundary pair. Four space boundary surface pairs which contained in the thermal zones, related to the slab among the ground and the first floor, are removed. In the GF space partition on the other hand, as it is displayed in parts IIA and IIB in Figure 9, the space boundaries referring to all internal wall partitions on the ground and first floors are removed. For this partition the neighbor external space boundaries referring to the same wall entity are merged and the all the space boundaries referring to the slab among the two floors are merged into one thermal zone boundary surface pair among the two zones. There are, however some adjacent external wall space boundaries in the ground floor visible in the back view in part IV in Figure 8 and in parts IB and IIB in Figure 9 which, although they belong to the same zone, are not merged because they are referring to different wall elements.

In order to demonstrate the simulation execution time reduction achieved by the proposed simplification method, the EnergyPlus ${ }^{\mathrm{TM}}$, was selected as the BEPS software. Four separate idf files (input data files of EnergyPlus) were automatically generated using the method presented in (Giannakis et al., 2015) and the respective zone boundary surface topologies, for the two proposed partition scenarios (vertical (EW) and the horizontal (GF)) and for two simplification methods. The examined simplification methods are: (a) simple surface re-indexing (SR) and (b)

\section{Vertical Partition}

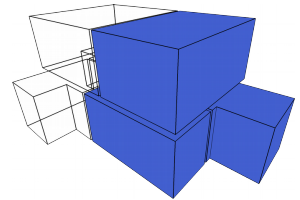

IA. East space volumes

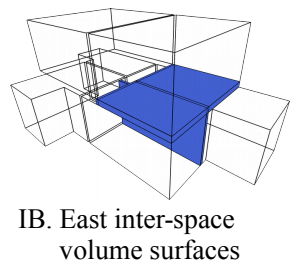

volume surfaces

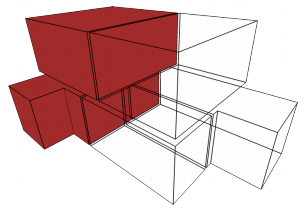

IC. West space volumes

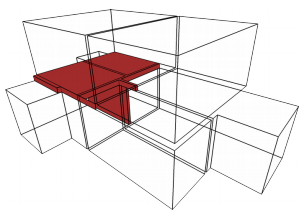

ID. West inter-space volume surfaces

\section{Horizontal Partition}

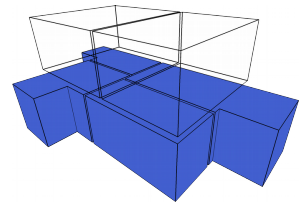

IIA. Ground space volumes

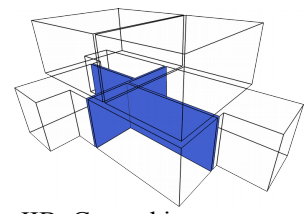

IIB. Ground inter-space volume surfaces

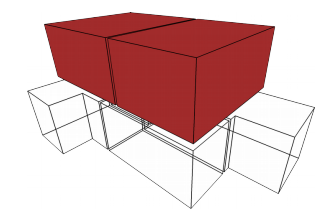

IIC. First floor space volumes

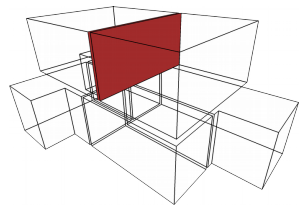
volume surfaces
IID. First floor inter-space

Figure 8: Zone space volumes and inter-space volume surfaces of the application scenarios.

\section{Vertical Partition}

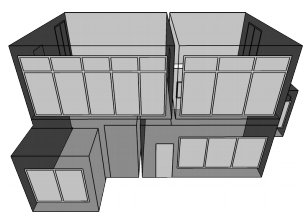

IA. Zone boundaries (front view)

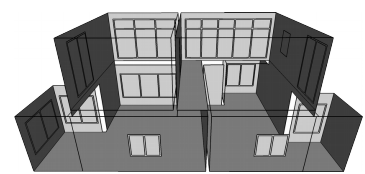

IB. Zone boundaries (rear view)

\section{Horizontal Partition}

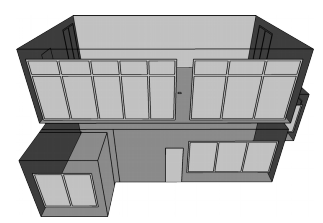

IIA. Zone boundaries (front view)

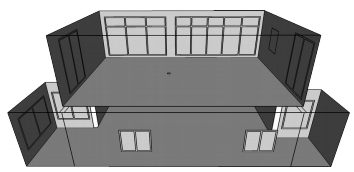

IIB. Zone boundaries (rear view)
Figure 9: Thermal zone boundaries for the two space partition scenarios (EW-left column) and (GF-right column). Back-face culling has been activated to render the building interior visible.

surface re-indexing and merging (SRM). The geometric content of the files referring to the SRM method for the two scenarios, is displayed by the Google SketchUp ${ }^{\mathrm{TM}}$ platform, in parts I (vertical partition) 
and II (horizontal partition) of Figure 10.

\section{Vertical Partition}

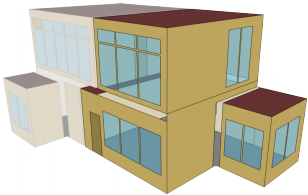

IA. Eastward zone

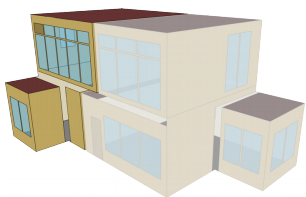

IB. Westward zone
II. Horizontal Partition

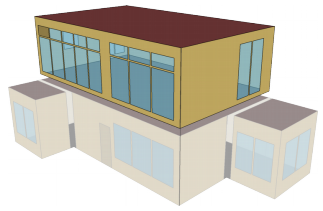

IIA. First floor zone

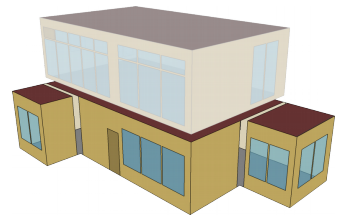

IIB. Fround floor zone
Figure 10: Visual representation of the geometric content of the IDF files for the two space partitions of the demonstration building.

No active (energy consuming) components were taken in to account during the simulations, which run for a whole year period for the same weather boundary conditions in all examined scenarios. The total execution times for the simplification methods (SR and SMR) and the partition scenarios (EW and GF) are reported in Table 1. The full model simulation run time for this demo building is 5.22 seconds. As it is expected, additional simulation run time reduction can be achieved when surface merging is considered (SRM method) for both partition scenarios. For the East-West partition the reduction drops further from a $17.24 \%$ drop in SR to a $24.71 \%$ in SRM and for the ground-first floor partition the decrease is from a $24.33 \%$ drop in SR to a $36.21 \%$ in SRM.

Table 1: Simulation run time results (in seconds). SR : Surface re-indexing

SRM : Surface re-indexing and merging

\begin{tabular}{|l|l|l|}
\hline Partition & SR & SRM \\
\hline EW & $4.32(\downarrow 17.24 \%)$ & $3.93(\downarrow 24.71 \%)$ \\
\hline GF & $3.95(\downarrow 24.33 \%)$ & $3.33(\downarrow 36.21 \%)$ \\
\hline
\end{tabular}

Full model execution time: 5.22

To examine the deviation of the simulation results of: the simplified model using surface re-indexing (SR) and the simplified model using surface re-indexing and merging (SRM), from the full model simulation (FM), the mean absolute error (MAE) is considered and is applied on the simulation output values of the total building energy demand. For the both models $\mathrm{SR}$ and SRM the MAE is calculated from:

$$
M A E_{S R}=\frac{1}{N} \sum_{i=1}^{N}\left|M_{i}-F M_{i}\right|
$$

where $M_{i}$ are the model under investigation samples $(M=S R$ or $M=S R M), F M_{i}$ are the full model samples and $\mathrm{N}$ is their total number. The two examined simplified models gave the following MAE values: $M A E_{S R}=0.5 \%$ and $M A E_{S R M}=1.1 \%$. The increase in the MAE values $\left(M A E_{S R M}>M A R_{S R}\right)$ when surface merging is included, can be justified by the fact that the surface area of the 2 a space boundary surfaces increases since the $2 \mathrm{~b}$ space boundary surfaces are absorbed and as a result the total building energy demand values also increase, diverging further from the full model's energy demand values.

\section{Conclusions}

A geometric simplification algorithm which achieves building energy performance simulation speedup by grouping spaces into zones and unifying space boundary surfaces into thermal zone boundaries, was introduced. The reduction of the total number of thermal zones (due to spaces-to-zones grouping) and the reduction of the total number of space boundary surfaces (due to space boundary surface merging) decreases the total simulation execution time, as the number of the differential equations solved and the number of boundary conditions taken into account by the simulation solver are decreased. To demonstrate the generality and robustness of the introduced algorithm an imaginary demonstration building was considered with internal building volumes are slightly offset. The algorithm is intended to be used together with the zone reduction process described in (Giannakis et al., 2017), to explore in an automated manner for large building complexes, trade offs between simulation run time reduction and loss of accuracy in the simulation results.

The introduced algorithm has two limitations which is a subject of further investigation. The first limitation is related to unnecessary holes sometimes appearing after the zone boundary merging stage, caused by gaps in the inter-space volumes of the zones, illustrated in part I of Figure 11. These holes do not disappear after the final zone boundary surface projection and intersection operations. Although these holes appear in the algorithms' output zone boundary surface topology, they are omitted in the geometric content of the BEPS model as they are not related to any building opening.

The second limitation is related to the fact that during the space-to-zone boundary surface merging stage (stage (c) in block diagram of Figure 2), the material layers of the building constructions which are related elements of adjacent space boundary surfaces, are not taken into account. By including the material layer sets of the building constructions into the merging stage, further reduction of the number of the space boundary surfaces can be achieved, since coplanar and adjacent space boundaries with the same relating spaces but with different related elements containing 
the same material layer set, could be unified. Such case is illustrated in part II of Figure 11.

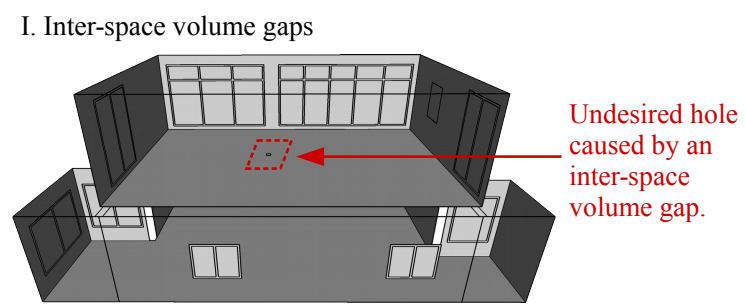

II. Unmerged space boundaries with the same material layer set.

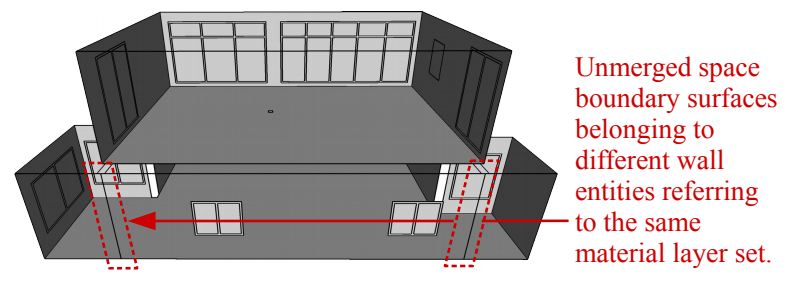

Figure 11: Example of an inter-space volume gap limitation.

Finally the algorithm is going to be tested in the future on large buildings where the benefits of simulation time reduction at the expense of acceptable loss in the simulation results accuracy, will become more evident. The algorithm could used as software component applied to simplify the baseline (spaces are treated as individual thermal zones) BEPS models, using the spaces to thermal zones grouping data, which are either included in the IFC BIM file or they are produced by automatic zoning algorithms.

\section{Acknowledgment}

The research leading to these results has been partially funded by the European Commission H2020EeB5-2015 project "Optimised Energy Efficient Design Platform for Refurbishment at District Level" under contract \#680676 (OptEEmAL).

\section{References}

Andriamamonjy, A., D. Saelens, and R. Klein (2018). An automated IFC-based workflow for building energy performance simulation with Modelica. Automation in Construction 91, 166-181.

ASHRAE 90.1 (2010). Energy standard for buildings except low-rise residential buildings. ASHRAE/IESNA Standard.

Georgescu, M. and I. Mezić (2015). Building energy modeling: A systematic approach to zoning and model reduction using koopman mode analysis. Energy and buildings 86, 794-802.

Giannakis, G., G. Kontes, I. Korolija, and D. Rovas (2017). Simulation-time reduction techniques for a retrofit planning tool. In Building Simulation Conference of IBPSA, San Fransisco, US.
Giannakis, G., G. N. Lilis, M. Garcia, G. Kontes, C. Valmaseda, and D. Rovas (2015). A methodology to automatically generate geometry inputs for energy performance simulation from IFC BIM models. In Building Simulation Conference of IBPSA, Hyderabad, India, pp. 504-511.

Giannakis, G., M. Pichler, G. Kontes, H. Schranzhofer, and D. Rovas (2013). Simulation speedup techniques for computationally demanding tasks. In Building Simulation Conference of IBPSA.

Lilis, G. N., G. I. Giannakis, and D. V. Rovas (2017). Automatic generation of second-level space boundary topology from IFC geometry inputs. Automation in Construction 76, 108-124.

Rose, C. M. and V. Bazjanac (2015). An algorithm to generate space boundaries for building energy simulation. Engineering with Computers 31(2), 271280 .

Thorade, M., J. Rädler, P. Remmen, T. Maile, R. Wimmer, J. Cao, M. Lauster, C. NytschGeusen, D. Müller, and C. van Treeck (2015). An open toolchain for generating modelica code from building information models. In Proceedings of the 11th International Modelica Conference, pp. 383391. Versailles, France, 21-23 September 2015.

Vatti, B. R. (1992). A generic solution to polygon clipping. Communications of the ACM 35(7), 5663.

Weise, M., T. Liebich, R. See, V. Bazjanac, T. Laine, and B. Welle (2011). Implementation guide: Space boundaries for energy analysis. General Services Administration (GSA) and Open Geospatial Consortium (OSC), 1-62.

Wimmer, R., J. Cao, P. Remmen, T. Maile, J. ODonnel, J. Frisch, R. Streblow, D. Müller, and C. van Treeck (2015). Implementation of advanced bimbased mapping rules for automated conversions to modelica. In Proceedings of the 14th IBPSA Building Simulation Conference, pp. 419-426. Hyderabad, India, 7-9 December 2015. 